\title{
ON DIOPHANTINE APPROXIMATIONS $\left({ }^{1}\right)$
}

\author{
BY \\ L. C. EGGAN( $\left({ }^{2}\right)$
}

1. Introduction. In 1891, A. Hurwitz [8] proved that for any irrational number $\theta$ there are infinitely many rational approximations $p / q$ which satisfy the inequality

$$
q^{2}|\theta-p / q|<5^{-1 / 2} \text {. }
$$

Although the constant $5^{-1 / 2}$ cannot be improved, it can be decreased if certain irrational numbers are removed from consideration. Let us say that $\theta$ is equivalent to $\theta^{\prime}$ in case there are integers $a, b, c, d$ such that

$$
\theta^{\prime}=(a \theta+b) /(c \theta+d) \text { with }|a d-b c|=1 .
$$

Then (1.1) can be improved thus: if the irrational number $\theta$ is not equivalent to $\left(5^{1 / 2}-1\right) / 2$, then there are infinitely many rational numbers $p / q$ such that

$$
q^{2}|\theta-p / q|<8^{-1 / 2}
$$

Now in case only one rational approximation $p / q$, and not infinitely many, is asked for, the constants in (1.1) and (1.2) can be improved. A. V. Prasad [21] showed in 1948 that to every irrational $\theta$ there is at least one rational approximation $p / q$ satisfying (1.1) with $5^{-1 / 2}$ replaced by the smaller value $2 /\left(3+5^{1 / 2}\right)$. We prove that $(1.2)$ can be improved by replacing $8^{-1 / 2}$ with $1 /\left(2^{1 / 2}+3 / 2\right)$ if only one approximation $p / q$ is required. In fact, in $\$ 2$ we prove much more. We answer the question: What is the best possible constant in an inequality of type (1.2) if we want at least $m$ rational approximations to an irrational number which has, in its continued fraction expansion, infinitely many partial quotients greater than or equal to $n$.

In $\S 3$ we consider other situations where there is a well-established theory of "infinitely many approximations," and we treat the corresponding problem of "at least one approximation." We note that in some of our results the constant is best possible. In $\S 4$, we extend a result of J. F. Koksma [10].

We conclude this section by stating some well known facts about continued fractions so that they will be available for easy reference and referral. We denote the continued fraction expansion of the irrational number $\theta$ by

Presented to the Society, September 1, 1960 under the title $A n$ extension of a theorem of A. V. Prasad; received by the editors July 17,1960 .

(1) This research was supported in part by the Office of Naval Research and in part by the National Science Foundation while the author was a N.S.F. Cooperative Graduate Fellow.

(2) This is a large part of the author's doctoral dissertation at the University of Oregon. The author wishes to express his gratitude to Professor Ivan Niven under whose direction the thesis was written. 


$$
\begin{aligned}
\theta & =a_{0}+\frac{1}{a_{1}+\frac{1}{a_{2}+.}} \\
& =a_{0}+\frac{1}{a_{1}+} \frac{1}{a_{2}+} \cdots=\left[a_{0}, a_{1}, a_{2}, \cdots\right],
\end{aligned}
$$

where each $a_{j}$ is an integer and $a_{j} \geqq 1$ for $j \geqq 1$. Let $\alpha_{n}=\left[a_{n+1}, a_{n+2}, \cdots\right]$, $\beta_{n}=\left[a_{n}, a_{n-1}, \cdots, a_{1}\right]$ and let $p_{n} / q_{n}$ denote the $n$th convergent to $\theta$. Then

$$
\theta-\frac{p_{n}}{q_{n}}=\frac{(-1)^{n}}{\left(\alpha_{n}+\frac{q_{n-1}}{q_{n}}\right) q_{n}^{2}}=\frac{(-1)^{n}}{\left(\alpha_{n}+\frac{1}{\beta_{n}}\right) q_{n}^{2}},
$$

and for $\sigma= \pm 1$,

$$
\left|\theta-\frac{p_{n}+\sigma p_{n-1}}{q_{n}+\sigma q_{n-1}}\right|=\frac{1}{\left(1+\frac{\sigma}{\alpha_{n}-\sigma}-\frac{\sigma}{\beta_{n}+\sigma}\right)\left(q_{n}+\sigma q_{n-1}\right)^{2}} .
$$

By using the definition of $p_{n} / q_{n}$, we have

$$
\theta=[0, n, n, \cdots] \text { implies } p_{j}=q_{j-1} \quad \text { for } j \geqq 0 .
$$

Finally we remark that two irrational numbers are equivalent if and only if their continued fraction expansions are the same from some point on.

2. An extension of a theorem of Prasad. Prasad [21] proved that for any irrational $\theta$, there are at least $m$ pairs of relatively prime integers $p, q, q>0$, satisfying

$$
|\theta-p / q| \leqq 1 /\left(c_{m} q^{2}\right)
$$

where $c_{m}=\left(5^{1 / 2}+1\right) / 2+h_{2 m-1} / k_{2 m-1}$, where $h_{j} / k_{j}$ is the $j$ th convergent to $\left(5^{1 / 2}-1\right) / 2$. Further, if $\theta=\left(5^{1 / 2}-1\right) / 2$, then there are exactly $m$ solutions. This section is devoted to a proof of an extension of this theorem of Prasad.

We first state a lemma the proof of which is routine computation.

Lemma 2.1. Let $n$ be a positive integer and let

$$
c=\left(\left(n^{2}+4\right)^{1 / 2}+n\right) / 2+1 / n .
$$

Then

(a)

$$
c<n+2 / n<n+1 /(n-1)+1 /(n+1) \quad \text { if } n \geqq 2,
$$

and 


$$
c<n+\frac{n-1}{n^{2}-n+1}+\frac{n+2}{n^{2}+2 n-1}
$$

if $n \geqq 3$.

Prasad showed that the constant in (2.1) cannot be improved in case $\theta=\left(5^{1 / 2}-1\right) / 2$. In fact, it is not hard to see that many irrational numbers, all equivalent to $\left(5^{1 / 2}-1\right) / 2$, also require this constant. What about those irrationals not equivalent to $\left(5^{1 / 2}-1\right) / 2$ ? Can the constant be improved for these irrationals? The affirmative answer is a consequence of the following theorem.

Theorem 2.2. Let $\theta_{2}=2^{1 / 2}-1$ and let $P_{j} / Q_{j}$ denote the $j$ th convergent to $\theta_{2}$. Then for any irrational $\theta$ not equivalent to $\left(5^{1 / 2}-1\right) / 2$ and any positive integer $m$, there are at least $m$ solutions in relatively prime integers $p, q, q>0$, to the inequality

$$
|\theta-p / q| \leqq 1 /\left(K_{m} q^{2}\right)
$$

where $K_{m}=2^{1 / 2}+1+P_{2 m-1} / Q_{2 m-1}$. Moreover, if $\theta=\theta_{2}$, there are exactly $m$ solutions.

Both this result and Prasad's theorem are special cases of the next theorem.

THEOREM 2.3. Let $n$ be a positive integer and let $\theta_{n}=[0, n, n, \cdots]$ $=\left(\left(n^{2}+4\right)^{1 / 2}-n\right) / 2$. For any positive integer $m$, let

$$
c_{m}=\theta_{n}+n+P_{2 m-1} / Q_{2 m-1}
$$

where $P_{j} / Q_{j}$ is the jth convergent to $\theta_{n}$. Then if $\theta=\left[a_{0}, a_{1}, a_{2}, \cdots\right]$ is irrational and if $a_{j} \geqq n$ for infinitely many values of $j$, there are at least $m$ solutions in relatively prime integers $p, q, q>0$, to the inequality

$$
|\theta-p / q| \leqq 1 /\left(c_{m} q^{2}\right) \text {. }
$$

Moreover, the constant $c_{m}$ cannot be improved, since for $\theta=\theta_{n}$ there are exactly $m$ solutions and equality is attained.

Proof. If $n=1$, then any irrational $\theta$ satisfies the hypothesis and the statement is precisely the theorem of Prasad. We assume therefore that $n \geqq 2$.

We first note that

$$
\begin{aligned}
c_{1} & =\left(\left(n^{2}+4\right)^{1 / 2}-n\right) / 2+n+1 / n \\
& =\left(\left(n^{2}+4\right)^{1 / 2}+n\right) / 2+1 / n,
\end{aligned}
$$

and since the odd convergents decrease to $\theta_{n}$,

$$
c_{1}>c_{2}>\cdots>\theta_{n}+n+\theta_{n}=\left(n^{2}+4\right)^{1 / 2} .
$$

Without loss of generality we may assume $0<\theta<1$. Let $\theta=\left[0, a_{1}, a_{2}, \cdots\right]$ be the simple continued fraction expansion of $\theta$ and let $p_{j} / q_{j}$ denote the $j$ th convergent to $\theta$. Let $\alpha_{j}=\left[a_{j+1}, a_{j+2}, \cdots\right]$ and $\beta_{j}=\left[a_{j}, a_{j-1}, \cdots, a_{1}\right]$ so that 


$$
\left|\theta-p_{j} / q_{j}\right|=1 /\left(A_{j} q_{j}^{2}\right)
$$

where

$$
A_{j}=\alpha_{j}+\frac{q_{j-1}}{q_{j}}=\alpha_{j}+\frac{1}{\beta_{j}},
$$

using (1.3). Thus we need only exhibit $m$ values of $j$ for which $A_{j} \geqq c_{m}$.

If $a_{j+1} \geqq n+1$ for infinitely many values of $j$, then by (2.6), (2.3), Lemma 2.1(a) and (2.4),

$$
A_{j}>\alpha_{j}>a_{j+1} \geqq n+1 \geqq n+2 / n>c_{1} \geqq c_{m} .
$$

Hence in this case there are infinitely many solutions to (2.2).

We may assume then that $a_{j} \leqq n$ for $j \geqq j^{\prime}$. We now break the argument into three parts. First we show that for $n=2$, if $\theta$ is not equivalent to $\theta_{2}$, then there are infinitely many solutions to (2.2). Next we show for $n \geqq 3$ that $\theta$ not equivalent to $\theta_{n}$ implies there are infinitely many solutions to (2.2). Finally, we show that if $n \geqq 2$, then $\theta$ equivalent to $\theta_{n}$ implies there are at least $m$ solutions to (2.2).

In the case $n=2$, if $\theta$ is not equivalent to $\theta_{2}$ then there must exist infinitely many $j \geqq j^{\prime}+2$ such that $a_{j+1}=2$ and $a_{j+2}=1$. For such a $j$ we have

$$
\begin{gathered}
A_{j}=\left[2,1, a_{j+3}, \cdots\right]+\left[0, a_{j}, a_{j-1}, \cdots, a_{1}\right] \\
1+\frac{1}{1+\frac{1}{2+1}}
\end{gathered}+\frac{1}{2+\frac{1}{1+\frac{1}{2+1}}}=2+\frac{72}{77} .
$$

Now $2+72 / 77>2^{1 / 2}+3 / 2=c_{1} \geqq c_{m}$ (for $\left.n=2\right)$. Thus in this case there are infinitely many solutions of (2.2).

To complete this second part where $n \geqq 3$, we require 4 cases.

Case 1. Suppose $a_{j} \leqq n-1, a_{j+1}=n, a_{j+2} \leqq n-1$ for infinitely many $j \geqq j^{\prime}$. Then for such a $j$,

$$
A_{j}>a_{j+1}+\frac{1}{a_{j+2}+1}+\frac{1}{a_{j}+1} \geqq n+\frac{1}{n}+\frac{1}{n}>c_{1},
$$

the last inequality by reason of Lemma 2.1(a). Therefore in this case we have infinitely many solutions to (2.2).

Case 2 . Suppose $a_{j+1}=n$ and $a_{j+2} \leqq n-2$ for infinitely many $j \geqq j^{\prime}$. Then for such a $j$,

$$
A_{j}>a_{j+1}+\frac{1}{a_{j+2}+1}+\frac{1}{a_{j}+1} \geqq n+\frac{1}{n-1}+\frac{1}{n+1}>c_{1},
$$


the last inequality again by reason of Lemma $2.1(\mathrm{a})$. We have infinitely many solutions to $(2.2)$ in this case also.

By considering Cases 1 and 2, we see that we may assume the existence of a $j^{\prime \prime}$ such that for $j \geqq j^{\prime \prime}$,

$$
a_{j+1}=n \text { and } a_{j+2} \leqq n-1 \text { imply } a_{j+2}=n-1 \text { and } a_{j}=n .
$$

Because of this last statement we see that we need only consider the following situations.

Case 3. Suppose $a_{j} \leqq n-1, a_{j+1}=a_{j+2}=n, a_{j+3}=n-1$ for infinitely many $j \geqq j^{\prime \prime}+2$. Then for such a $j$

$$
\begin{aligned}
A_{j} & >a_{j+1}+\frac{1}{a_{j+2}+} \frac{1}{a_{j+3}}+\frac{1}{a_{j}+} \frac{1}{a_{j-1}}+\frac{1}{a_{j-2}+1} \\
& \geqq n+\frac{1}{n+} \frac{1}{n-1}+\frac{1}{n-1+} \frac{1}{1+} \frac{1}{n+1} \\
& =n+(n-1) /\left(n^{2}-n+1\right)+(n+2) /\left(n^{2}+2 n-1\right) \\
& >c_{1},
\end{aligned}
$$

using Lemma 2.1 (b). And again we have infinitely many solutions to (2.2).

Case 4. Suppose $a_{j-1}=a_{j}=a_{j+1}=n$ and $a_{j+2}=n-1$ for infinitely many $j \geqq j^{\prime \prime}$. For such a $j$

$$
\begin{aligned}
A_{j}> & {\left[a_{j+1}, a_{j+2}, a_{j+3}, a_{j+4}+1\right]+1 /\left[a_{j}, a_{j-1}\right] } \\
& \geqq[n, n-1,1, n+1]+1 /[n, n] \\
& >n+(n+2) /\left(n^{2}+2 n-1\right)+(n-1) /\left(n^{2}-n+1\right) \\
& >c_{1},
\end{aligned}
$$

again by use of Lemma 2.1 (b). Hence in this case also we have infinitely many solutions to (2.2) for any value of $m$.

Thus we see that for our final part, for any $n \geqq 2$ we may assume that $a_{j}=n$ from some point on. Let $J$ be the smallest integer for which

$$
a_{j}=n, \quad \text { if } j>J \text {. }
$$

We now consider three cases.

Case $1^{\circ}$. Suppose $a_{J} \geqq n+1$. We will show that for the $m$ values of $j$, $j=J-1, J+1, \cdots, J+2 m-3$,

$$
A_{j} \geqq c_{m} .
$$

For $j=J-1$,

$$
A_{j}>a_{J} \geqq n+1>c_{1} \geqq c_{m}
$$

by use of Lemma 2.1(a). For any other values of $j$, 


$$
\alpha_{j}=[n, n, n, \cdots]=n+\theta_{n} .
$$

Also

$$
1 / \beta_{j}=\frac{1}{n+} \cdots+\frac{1}{a_{J}+} \cdots+\frac{1}{a_{1}}
$$

where there are $j-J$ occurrences of $n$ preceding $a_{J}$. Since $j-J$ is odd and $a_{J} \geqq n+1$, we will decrease the above expression if we replace

$$
1 /\left[a_{J}, \cdots, a_{1}\right]
$$

by the larger quantity $1 /(n+1 / n)$. Hence we obtain

$$
A_{j}>n+\theta_{n}+1 /[n, n, \cdots, n]=n+\theta_{n}+P_{j-J+2} / Q_{j-J+2} .
$$

Thus $A_{j} \geqq c_{m}$ since $j-J+2$ is odd and $\leqq 2 m-1$. We have $m$ solutions to (2.2) which completes the proof of Case $1^{\circ}$.

Case $2^{\circ}$. Suppose $a_{J} \leqq n-1$. We will show that $A_{j} \geqq c_{m}$ for at least $m$ values of $j$, namely for

$$
j=J, J+2, \cdots, J+2 m-2 .
$$

First, for $j=J$,

$$
\begin{aligned}
A_{j} & =\left[a_{J+1}, \cdots\right]+1 /\left[a_{J}, \cdots, a_{1}\right] \geqq \theta_{n}+n+1 /\left(a_{J}+1\right) \\
& \geqq \theta_{n}+n+1 / n=c_{1} \geqq c_{m},
\end{aligned}
$$

by use of (2.7). For any of the other $m-1$ values of $j$,

$$
1 / \beta_{j}=\frac{1}{n+} \cdots+\frac{1}{a_{J}+} \cdots+\frac{1}{a_{1}}
$$

where $a_{J}$ is preceded by $j-J$ occurrences of $n$. In this case $a_{J} \leqq n-1$ so

$$
1 /\left[a_{J}, \cdots, a_{1}\right] \geqq 1 / n \text {. }
$$

Hence, since $j-J$ is even,

$$
1 / \beta_{j} \geqq[0, n, \cdots, n]=P_{j-\checkmark+1} / Q_{j-\checkmark+1}
$$

where, as indicated, the number of $n$ 's is $j-J+1$. This together with (2.7) gives us

$$
A_{j} \geqq \theta_{n}+n+P_{j-J+1} / Q_{j-J+1} .
$$

Thus, as before, $A_{j} \geqq c_{m}$ since $j-J+1$ is odd and $\leqq 2 m-1$.

Finally we consider

Case $3^{\circ}$. Suppose $J=0$ so that $\theta=\theta_{n}$. Then for

$$
j=1,3, \cdots, 2 m-1 \text {, }
$$

we have 


$$
\begin{aligned}
A_{j} & =\left[a_{j+1}, \cdots\right]+Q_{j-1} / Q_{j} \\
& =n+\theta_{n}+P_{j} / Q_{j}(\text { using }(2.7) \text { and }(1.5)) \\
& =c_{(j+1) / 2} \geqq c_{m} .
\end{aligned}
$$

The last inequality follows since $j \leqq 2 m-1$ and odd.

To show that the constant in (2.2) cannot be improved we use a theorem due to Legendre (see [5, Theorem 184]) which implies that, since $c_{m}>\left(n^{2}+4\right)^{1 / 2}$ $>2$, any solution of (2.2) must be a convergent of $\theta$. However, for $\theta=\theta_{n}$, the only $P_{j} / Q_{j}$ for which $A_{j} \geqq c_{m}$ are those stated in Case $3^{\circ}$, since $P_{j} / Q_{j}$ $<P_{2 m-1} / Q_{2 m-1}$ if $j$ is even or if $j$ is odd and greater than $2 m-1$. This completes the proof of the theorem.

There are a number of corollaries to the preceding theorem. The first corresponds to the second result in the Markoff chain (see, for example, Cassels $[2$, p. 18]) and is a special case of Theorem 2.2.

CoRollary 2.4. If $\theta$ is not equivalent to $\left(5^{1 / 2}-1\right) / 2$, then there is at least one pair of relatively prime integers $p, q, q>0$, satisfying

$$
|\theta-p / q| \leqq 1 /\left(\left(2^{1 / 2}+3 / 2\right) q^{2}\right) .
$$

Moreover, for $\theta=2^{1 / 2}-1$ there is precisely one pair.

Although the following result is well-known $\left({ }^{3}\right)$, it is interesting to note that it follows immediately from (2.4) and Theorem 2.3.

Corollary 2.5 (Perron). For any positive integer $n$, if $\theta$ is as in Theorem 2.3 , then there are infinitely many pairs of relatively prime integers $p, q, q>0$, satisfying

$$
|\theta-p / q|<1 /\left(\left(n^{2}+4\right)^{1 / 2} q^{2}\right) .
$$

Moreover, for $\theta=\theta_{n}$ the constant $\left(n^{2}+4\right)^{1 / 2}$ cannot be increased.

We are able, as a consequence of the proof of Theorem 2.3 , to improve the constant in Corollary 2.5 for all but a countable number of relevant $\theta .\left(^{4}\right)$

Corollary 2.6. For any positive integer $n$, if $\theta$ is as in Theorem 2.3 but $\theta$ is not equivalent to $\theta_{n}$, then there are infinitely many pairs of relatively prime integers $p, q, q>0$, satisfying

$$
\left|\theta-\frac{p}{q}\right|<\frac{1}{\left(\frac{\left(n^{2}+4\right)^{1 / 2}+n}{2}+\frac{1}{n}\right) q^{2}} .
$$

(8) See J. F. Koksma, Diophantische Approximationen, Ergebnisse Math. u. ihrer Grenzgebiete 4, Berlin, Springer (or New York, Chelsea), 1936, p. 33, Satz 17 or [14, Satz 9]. Obrechkoff [18] has proved a stronger result.

(4) It should be remarked that $\mathrm{K}$. Shibata, in On the order of the approximation of irrational numbers by rational numbers, Tohoku Math. J. vol. 30 (1929) pp. 22-50, has shown that the best coefficient of $q^{2}$ in this case is $O\left(1 / n^{2}\right)$ greater than $n+2 / n$. 
3. One approximation in some other settings. As we noted in the preceding section, Prasad considered the question of one approximation corresponding to the Hurwitz theorem. There are other theorems stating the existence of infinitely many approximations about which the question of one approximation may be asked. In this section we consider three such theorems.

Let us denote by $(k)$ the inequality

$$
|\theta-p / q|<k / q^{2} \text {. }
$$

Scott [24] showed that if we restrict the fractions $p / q$ to be from any one of the three classes (i) $p, q$ both odd, (ii) $p$ odd, $q$ even, or (iii) $p$ even, $q$ odd, then there are infinitely many such $p / q$ satisfying (1). Other proofs of this result have been given by Robinson [22], Oppenheim [20] and Kuipers and Meulenbeld [11]. Robinson also showed that if any pair of these classes were used, there would be infinitely many satisfying (1/2). Here, then, are two results about which we can ask if we can improve the constants 1 and $1 / 2$ if we wish only one solution to the inequality. The answer is contained in the following theorem.

THEOREM 3.1. Let $\theta$ be an irrational number and consider the three types of fractions odd/odd, odd/even, and even/odd.

(a) If any one of the three types is chosen, then there is at least one fraction $p / q$ of the chosen type satisfying

$$
|\theta-p / q|<1 / q^{2} \text {. }
$$

Moreover, the constant 1 cannot be diminished.

(b) If any two of the three types are chosen, then there is at least one fraction $p / q$ of one of the chosen types satisfying

$$
|\theta-p / q|<1 / 2 q^{2}
$$

Morever, the constant 2 cannot be increased.

The first statement in each of (a) and (b) follows trivially from the theorems of Scott and Robinson. The last statements follow from the following two theorems. An easy modification of an example given by Oppenheim [20] yields the examples in the proof of Theorem 3.2, whereas the examples in Theorem 3.3 are due to Robinson [22].

THEOREM 3.2. For any $\sigma>0$ and for any choice of one of the three types odd/odd, odd/even, or even/odd, there is an irrational number $\theta$ so that no fraction of the chosen type satisfies

$$
|\theta-p / q| \leqq(1-\sigma) / q^{2} \text {. }
$$

Proof. Let $\sigma>0$ be given and choose an integer $m$ so that $m>1 / \sigma$. Let $\theta=\left[a_{0}, a_{1}, a_{2}, \cdots\right]$ where each $a_{n} \geqq 2 m+1(n \geqq 0)$. If odd/odd is the chosen type, further require each $a_{n}$ to be even. If even/odd was chosen, let $a_{0}$ be 
odd and $a_{n}$ even for $n \geqq 1$. Finally, if odd/even was chosen, let $a_{0}$ and $a_{1}$ be odd and $a_{n}$ even for $n \geqq 2$. It is routine to check that for these choices of $\theta$, no convergent is of the chosen type.

Now let

$$
x=q^{2}|\theta-p / q| \text {. }
$$

If $x \geqq 1$, there is nothing to prove. If $x<1$, then $p / q$ must be a convergent or a secondary convergent (cf. Oppenheim [20] or Robinson [22]). Now by the choice of $\theta$, no convergent is of the chosen type. Hence we must have that $p / q$ is a secondary convergent, i.e.,

$$
p / q=\left(p_{n}+\mu p_{n-1}\right) /\left(q_{n}+\mu q_{n-1}\right) \quad(\mu= \pm 1)
$$

for some integer $n$, where $p_{n} / q_{n}$ is a convergent to $\theta$. Now if $n \geqq 1$, let $\alpha=\left[a_{n+1}, a_{n+2}, \cdots\right]$ and $\beta=\left[a_{n}, \cdots, a_{1}\right]$. Then by (1.4) we have

$$
\begin{aligned}
x & =\left(q_{n}+\mu q_{n-1}\right)^{2}\left|\theta-\left(p_{n}+\mu p_{n-1}\right) /\left(q_{n}+\mu q_{n-1}\right)\right| \\
& =(1+\mu /(\alpha-\mu)-\mu /(\beta+\mu))^{-1} \\
& =(\alpha-\mu)(\beta+\mu) /(\alpha \beta+1) \\
& =1-(2-\mu(\alpha-\beta)) /(\alpha \beta+1)>1-(2+\alpha+\beta) /(\alpha \beta+1) .
\end{aligned}
$$

Now

$$
\begin{aligned}
(\alpha \beta+1)-m(2+\alpha+\beta) & =(\alpha-m)(\beta-m)-m^{2}-2 m+1 \\
& >(m+1)^{2}-m^{2}-2 m+1>0,
\end{aligned}
$$

using the fact that $\alpha, \beta>2 m+1$. Hence

$$
x>1-(2+\alpha+\beta) / m(2+\alpha+\beta)=1-1 / m>1-\sigma .
$$

If $n=0$, then $x>1$ for $\mu=-1$. Thus

$$
p=p_{0}+p_{-1}=a_{0}+1 \text { and } q=q_{0}=1
$$

so

$$
\begin{aligned}
x & =\left|\theta-\left(a_{0}+1\right)\right|=1-\left[0, a_{1}, a_{2}, \cdots\right] \\
& >1-1 / m>1-\sigma .
\end{aligned}
$$

This completes the proof of Theorem 3.2 and consequently of Theorem 3.1(a).

THEOREM 3.3. For any $\sigma>0$ and for any choice of two of the three types odd/odd, odd/even, or even/odd, there is an irrational number $\theta$ so that no fraction of the chosen types satisfies

$$
|\theta-p / q| \leqq 1 /\left((2+\sigma) q^{2}\right) .
$$

Proof. Let $\sigma>0$ be given and choose $m>2 / \sigma$. Again by Legendre's theorem we need only consider convergents $p_{n} / q_{n}$ to $\theta=\left[a_{0}, a_{1}, \cdots\right]$. If odd/odd 
is not chosen, let $a_{0}$ be odd and greater than $m$, let $a_{2 n}=2$ for $n \geqq 1$, and let $a_{2 n-1}$ be even and greater than $m$ for $n \geqq 1$. Then $p_{2 n+1} / q_{2 n+1}$ is odd/even and $p_{2 n} / q_{2 n}$ is odd/odd so we need only consider odd convergents. But for $n \geqq 1$,

$$
\begin{aligned}
q_{2 n-1}\left|q_{2 n-1} \theta-p_{2 n-1}\right| & =\left(\left[a_{2 n}, a_{2 n+1}, \cdots\right]+\left[0, a_{2 n-1}, \cdots\right]\right)^{-1} \\
& >\left(a_{2 n}+1 / a_{2 n+1}+1 / a_{2 n-1}\right)^{-1} \\
& >(2+1 / m+1 / m)^{-1} \\
& >(2+\sigma)^{-1} .
\end{aligned}
$$

Thus the result is established if odd/odd is not chosen.

If even/odd is not chosen, let $a_{2 n}=2$ and $a_{2 n+1}$ be even and greater than $m$ for $n \geqq 0$. Then $p_{2 n} / q_{2 n}$ is even/odd and $p_{2 n+1} / q_{2 n+1}$ is odd/even so by (3.1) the result also follows in this case.

Finally, if odd/even is not chosen, let $a_{2 n+1}=2$ and $a_{2 n}$ be even and greater than $m$ for $n \geqq 0$. Then, as above, $p_{2 n} / q_{2 n}$ is even/odd and $p_{2 n+1} / q_{2 n+1}$ is odd/even. By an argument similar to that in (3.1) we obtain the result in this case. The proof of the theorem is thus complete.

In 1945, Segre [25] proved the following theorem:

Every irrational $\theta$ has infinitely many rational approximations $u / v$ such that

$$
\frac{-\gamma}{(1+4 \gamma)^{1 / 2} v^{2}}<\theta-\frac{u}{v}<\frac{1}{(1+4 \gamma)^{1 / 2} v^{2}}
$$

where $\gamma \geqq 0$ is arbitrary.

(Note that any even convergent satisfies the result for $\boldsymbol{\gamma}=0$ while for $\gamma=1$ the result is the classical theorem of Hurwitz.) Since that time a number of people, including Olds [19], Negoescu [16;17], Robinson [23], LeVeque [12] and Tornheim [27], have considered this problem of asymmetric approximation. Robinson showed further that for any $\sigma>0$ and any irrational $\theta$, the inequality

$$
\frac{-1}{\left(5^{1 / 2}-\sigma\right) q^{2}}<\frac{p}{q}-\theta<\frac{1}{\left(5^{1 / 2}+1\right) q^{2}}
$$

has infinitely many solutions. Thus one side of Hurwitz's theorem can be strengthened without essentially weakening the other.

Are there inequalities of the above form when only one solution is desired? In particular, is it possible to strengthen one side of Prasad's theorem without essentially weakening the other? The answer to these questions in the negative is contained in the following theorem.

THEOREM 3.4. Any statement of the form: "For any irrational $\theta$, there is at least one pair of integers $p, q$ so that

$$
-c / q^{2} \leqq p / q-\theta \leqq d / q^{2}
$$

for some $d, 0 \leqq d<\left(3-5^{1 / 2}\right) / 2, "$ is false unless $c>1 / 5^{1 / 2}$. 
Note 1. If $\theta$ does not satisfy the above statement, then $-\theta$ does not satisfy the corresponding statement with $c$ and $d$ interchanged.

Note 2. There is a true statement of the form given in the theorem for $c>1 / 5^{1 / 2}$, namely the result of Robinson quoted above.

Proof. Again we need only consider convergents to $\theta$. If $\theta=\left(5^{1 / 2}-1\right) / 2$, then all the odd convergents satisfy

$$
p / q-\theta \geqq\left(3-5^{1 / 2}\right) / 2 q^{2},
$$

and all the even convergents satisfy

$$
\theta-p / q>1 /\left(5^{1 / 2} q^{2}\right)
$$

The first inequality follows from the last two paragraphs of the proof of Theorem 2.3 (take $m=n=1$ ) and the second follows from

$$
\begin{aligned}
\theta-\frac{p_{2 n}}{q_{2 n}} & =\frac{1}{\left(1+\left(5^{1 / 2}-1\right) / 2+p_{2 n} / q_{2 n}\right) q_{2 n}^{2}} \\
& >\frac{1}{\left(\left(5^{1 / 2}+1\right) / 2+\left(5^{1 / 2}-1\right) / 2\right) q_{2 n}^{2}} \\
& =\frac{1}{5^{1 / 2} q_{2 n}^{2}},
\end{aligned}
$$

where we use (2.8) to obtain the first exhibited equality.

Let us now turn our attention to nonhomogeneous approximations; that is, to quantities of the form

$$
|\tau(v \theta-u-\alpha)|
$$

where $\theta$ is irrational, $\alpha$ is real and $u$ and $v$ are integers. Note that we have been considering quantities of this form for $\alpha=0$, the homogeneous case.

Before continuing, we require a notion of equivalence, first defined by Descombes [4], of ordered pairs of real numbers.

Definition 3.5. The pairs $(\theta, \alpha)$ and $\left(\theta^{\prime}, \alpha^{\prime}\right)$ are called equivalent, denoted

$$
(\theta, \alpha) \sim\left(\theta^{\prime}, \alpha^{\prime}\right)
$$

if there exists six integers $A, B, C, D, E, F$, with $A D-B C= \pm 1$, so that

$$
\theta^{\prime}=\frac{A \theta+B}{C \theta+D}, \quad \alpha^{\prime}=\frac{(A D-B C) \alpha}{C \theta+D}+\frac{E \theta+F}{C \theta+D}, \quad C \theta+D>0 .
$$

It is easy to verify that this relation is an equivalence relation. Note also that $(\theta, \alpha) \sim\left(\theta^{\prime}, \alpha^{\prime}\right)$ implies that $\theta$ is equivalent to $\theta^{\prime}$ under the definition given in $\$ 1$.

The paper of Descombes [4] is concerned with the proof of a theorem of which the following is a part. 
TheOREM 3.6. If $\theta$ is any irrational number, $\alpha$ any real number and $\sigma$ is greater than 0 :

(a) there exists an infinity of pairs of integers $(u, v)$, with $v>0$, such that

$$
v|v \theta-u-\alpha|<1 /\left(5^{1 / 2}\right)+\sigma,
$$

the constant $1 / 5^{1 / 2}$ cannot be diminished for the pairs $(\theta, \alpha)$ equivalent to

$$
\left(\frac{1+5^{1 / 2}}{2}, 0\right) \text {; }
$$

(b) if the pair $(\theta, \alpha)$ is different from these latter special pairs, there is an infinity of pairs of integers $(u, v)$, with $v>0$, such that

$$
v|v \theta-u-\alpha|<27 /\left(28\left(7^{1 / 2}\right)\right)+\sigma,
$$

the constant $27 /\left(28\left(7^{1 / 2}\right)\right)$ cannot be diminished for the pairs $(\theta, \alpha)$ equivalent to

$$
\left(\frac{7-7^{1 / 2}}{14}, \frac{1}{14}\right)
$$

(c) if the pair $(\theta, \alpha)$ is different at the same time from the special pairs of (a) and from the special pairs of (b), there exists an infinity of pairs of integers $(u, v)$, with $v>0$, such that

$$
v|v \theta-u-\alpha|<1 /\left(8^{1 / 2}\right)+\sigma,
$$

the constant $1 / 8^{1 / 2}$ cannot be diminished for the pairs $(\theta, \alpha)$ equivalent to

$$
\left(2^{1 / 2}, 0\right) \text {. }
$$
[1].

Part (a) of Theorem 3.6 is due to Khintchine [9] and part (b) to Cassels

Suppose we ask the question which Prasad asked in the homogeneous case. Can the constant $1 / 5^{1 / 2}$ in part (a) of the theorem be decreased if we wish only one solution? We do not know the solution to this question $\left({ }^{5}\right)$. However, the following is a partial result.

THEOREM 3.7. For any irrational $\theta$, any real $\alpha$ and any $\sigma>0$, there is at least one pair of integers $(u, v)$, with $v>0$, so that

$$
0<v|v \theta-u-\alpha|<1 /\left(5^{1 / 2}\right)+\sigma .
$$

Moreover, the constant $1 / 5^{1 / 2}$ cannot be replaced by any smaller number.

Proof. The first statement is an obvious corollary of Theorem 3.6(a).

(b) Note that the above together with Prasad's theorem (Theorem 2.3 with $n=1$ ) implies that the best constant $c$ for which there is at least one solution to $v|v \theta-u-\alpha|\langle c+\sigma$ is such that $2 /\left(5^{1 / 2}+3\right) \leqq c \leqq 1 / 5^{1 / 2}$. The corresponding question when $v$ may be negative was handled by P. Kanagasabapathy, Proc. Cambridge Philos. Soc. vol. 48 (1952) pp. 365-366. 
Descombes [4, especially p. 262] shows that if $\theta=\alpha$, we need only consider pairs $(u, v)$ equal to $\left(p_{n}, q_{n}+1\right)$, where $p_{n} / q_{n}$ is the $n$th convergent to $\theta$. (See also Sós [26] for a different derivation.) Thus let

$$
\alpha=\theta=\left(5^{1 / 2}-1\right) / 2=[0,1,1,1, \cdots] .
$$

Then for $v=q_{n}+1, u=p_{n}$, by the special character of $\theta$ (in particular, using (1.3), (2.8) and (1.5)),

$$
\begin{aligned}
v|v \theta-u-\alpha| & =\left(q_{n}+1\right)\left|q_{n} \theta-p_{n}\right| \\
& =\frac{q_{n}+1}{q_{n}(\theta+1)+q_{n-1}} .
\end{aligned}
$$

Now by (3.2), for $n$ even

$$
q_{n}\left|q_{n} \theta-p_{n}\right|>1 / 5^{1 / 2}
$$

so certainly the quantity in (3.3) is greater than $1 / 5^{1 / 2}$. Thus it suffices to prove that for $n$ odd

$$
\frac{q_{n}+1}{q_{n}(\theta+1)+q_{n-1}}>\frac{1}{5^{1 / 2}} .
$$

We do this by noting that the following statements are equivalent:

$$
\begin{aligned}
&\left(q_{n}+1\right) /\left(q_{n}(\theta+1)+q_{n-1}\right)>1 / 5^{1 / 2}, \\
& 5^{1 / 2} q_{n}+5^{1 / 2}>q_{n}(\theta+1)+q_{n-1}, \\
&\left(5^{1 / 2}-1-\theta\right) q_{n}+5^{1 / 2}>q_{n-1}, \\
& \theta q_{n}+5^{1 / 2}>q_{n-1}\left(\text { since } \theta=\left(5^{1 / 2}-1\right) / 2\right), \\
& \theta+\frac{5^{1 / 2}}{q_{n}}>\frac{q_{n-1}}{q_{n}}=\frac{p_{n}}{q_{n}}(\text { by }(1.5)), \\
&\left.\frac{5^{1 / 2}}{q_{n}}>\frac{p_{n}}{q_{n}}-\theta=\frac{1}{\left(\theta+1+q_{n-1} / q_{n}\right) q_{n}^{2}} \text { (since } n \text { is odd and by }(3.3)\right), \\
& 5^{1 / 2}>1 / q_{n}\left((\theta+1)+q_{n-1} / q_{n}\right) .
\end{aligned}
$$

But $q_{n} \geqq 1$ and $\theta+1+q_{n-1} / q_{n}>1$ so (3.4) holds.

This completes the proof of the theorem.

It should be remarked that the restriction

$$
v|v \theta-u-\alpha|>0
$$

is essential for our proof as this quantity is 0 for our choice of $\theta$ and $\alpha$ when $v=1$ and $u=0$.

4. An extension of a theorem of Koksma. Koksma [10], improving on a result of Hartman [6], showed by using part (a) of Theorem 3.6 that: 
If $\theta$ is irrational and $\sigma>0$, then for any integers $s \geqq 1, a, b$, there are infinitely many pairs of integers $p, q,(q>0)$, satisfying the conditions

$$
|\theta-p / q|<s^{2}(1+\sigma) /\left(5^{1 / 2} q^{2}\right)
$$

and

$$
p \equiv a(\bmod s), \quad q \equiv b(\bmod s) .
$$

We use parts (b) and (c) of Theorem 3.6 to show what happens if we disregard those numbers which require $5^{1 / 2}$ in the above result.

THEOREM 4.1. Let $\theta$ be any irrational number not equivalent to $\left(5^{1 / 2}-1\right) / 2$ and let $\sigma>0$. Then for any integers $s \geqq 1, a, b$, there are infinitely many pairs of integers $p, q,(q>0)$, so that

$$
|\theta-p / q|<s^{2}(27+\sigma) /\left(28\left(7^{1 / 2}\right) q^{2}\right)
$$

and

$$
p \equiv a(\bmod s), \quad q \equiv b(\bmod s) .
$$

Moreover, if $\theta$ is also not equivalent to $\left(7-7^{1 / 2}\right) / 14$ or if $s \neq 0(\bmod 14)$, then there are infinitely many pairs of integers $p, q,(q>0)$, satisfying (4.2) and

$$
|\theta-p / q|<s^{2}(1+\sigma) /\left(\left(8^{1 / 2}\right) q^{2}\right) \text {. }
$$

Proof. Let $\theta$ be irrational and not equivalent to $\left(5^{1 / 2}-1\right) / 2$, let $\sigma>0$ be given, and suppose $s \geqq 1, a, b$ are integers. By Theorem 3.6(b), there exist infinitely many pairs of integers $u, v$, with $v>0$, satisfying

$$
v|v \theta-u-\alpha|<\left(27+\sigma_{1}\right) /\left(28\left(7^{1 / 2}\right)\right)
$$

where $\alpha=(a-b \theta) / s$ and

$$
0<\sigma_{1}<\sigma-b \sigma(\sigma+27) /((\sigma+27) b+\sigma) .
$$

Let

$$
p=a+u s, \quad q=b+v s
$$

so

$$
\begin{aligned}
\theta q-p & =\theta(b+v s)-(a+u s) \\
& =s(v \theta-u-(a-b \theta) / s) \\
& =s(v \theta-u-\alpha) .
\end{aligned}
$$

Hence $|\theta q-p|=s|v \theta-u-\alpha|<s\left(27+\sigma_{1}\right) /\left(28\left(7^{1 / 2}\right) v\right)$ for infinitely many values of $v$. We therefore have infinitely many pairs of integers $p, q,(q>0)$, which satisfy (4.1) and (4.2), since for all $v$ so large that $q>((27+\sigma) b+\sigma) / \sigma$ we have 


$$
|\theta-p / q|<\frac{s\left(27+\sigma_{1}\right)}{28\left(7^{1 / 2}\right) q v}=\frac{s^{2}\left(27+\sigma_{1}\right)}{28\left(7^{1 / 2}\right) q(q-b)}<\frac{s^{2}(27+\sigma)}{28\left(7^{1 / 2}\right) q^{2}} .
$$

Now if

$$
(\theta,(a-b \theta) / s) \times\left(\left(7-7^{1 / 2}\right) / 14,1 / 14\right)
$$

then by part (c) of Theorem 3.6, the constant $27 /\left(28\left(7^{1 / 2}\right)\right)$ can be improved. The first condition in Definition 3.5 of the equivalence of pairs shows that if (4.4) does not hold, then $\theta$ is equivalent to $\left(7-7^{1 / 2}\right) / 14$. However, even if $\theta$ and $\left(7-7^{1 / 2}\right) / 14$ are equivalent, the second condition requires $s / 14$ to be an integer. Hence, if either of these two conditions is not satisfied, then there are infinitely many pairs of integers $u, v,(v>0)$ such that

$$
v|\theta v-u-(a-\theta b) / s|<\left(1+\sigma_{1}\right) / 8^{1 / 2}
$$

where $0<\sigma_{1}<\sigma-(\sigma+1) b \sigma /((\sigma+1) b+\sigma)$. Thus for

$$
p=a+u s, \quad q=b+v s
$$

and $v$ such that $q>1+(\sigma+1) b / \sigma$, we obtain infinitely many solutions to (4.3) and (4.2).

\section{BIBLIOGRAPHY}

1. J.W.S. Cassels, Über lim inf $\ln _{x \rightarrow+\infty} x|\theta x+\alpha-y|$, Math. Ann. vol. 127 (1954) pp. 288-304.

2. - An introduction to Diophantine approximations, Cambridge Tracts No. 45, Cambridge, 1957.

3. J. H. H. Chalk, Rational approximations in the complex plane. II, J. London Math. Soc. vol. 31 (1956) pp. 216-221.

4. R. Descombes, Sur la répartition des sommets d'une ligne polygonale régulière non fermée, Ann. Sci. Ecole Norm. Sup. vol. 73 (1956) pp. 283-355.

5. G. H. Hardy and E. M. Wright, The theory of numbers, 3rd ed., Oxford, Clarendon Press, 1954.

6. S. Hartman, Sur une condition supplementaire dans les approximations diophantiques, Colloq. Math. vol. 2 (1949) pp. 48-51.

7. E. Hlawka, Über die Approximation von zwei komplexen inhomogenen Linearformen, Monatsh. Math. vol. 46 (1937-1938) pp. 324-334.

8. A. Hurwitz, Über die angenäherte Darstellung der Irrationalzahlen durch rationale Brüche, Math. Ann. vol. 39 (1891) pp. 279-284.

9. A. Khintchine, Neuer Beweis und Verallgemeinerung eines Hurwitzschen Satzes, Math. Ann. vol. 111 (1935) pp. 631-637.

10. J. F. Koksma, Sur l'approximation des nombres irrationels sous une condition supplémentaire, Simon Stevin vol. 28 (1951) pp. 199-202.

11. L. Kuipers and B. Meulenbeld, Some properties of continued fractions, Acta Math. vol. 87 (1952) pp. 4-12. $1-6$.

12. W. J. LeVeque, On asymmetric approximations, Michigan Math. J. vol. 2 (1953) pp.

13. K. Mahler, On the product of two complex linear polynomials in two variables, J. London Math. Soc. vol. 15 (1940) pp. 213-236. 
14. M. Müller, Über die Approximation reeler Zahlen durch die Näherungsbritche ihres regelmässigen Kettenbruches, Arch. Math. vol. 6 (1955) pp. 253-258. 1956.

15. Ivan Niven, Irrational numbers, Carus Monograph No. 11, New York, John Wiley,

16. Nicolae Negoescu, Quelques precisions concernant le theorème de $M$. B. Segre sur des approximations asymetriques des nombres irrationnels par les rationnels, Bull. Ecole Polytech. Jassy, vol. 3 (1948) pp. 3-16.

17. - Note on a theorem of unsymmetric approximation, Acad. R. P. Romine Bul. Şti. Sect. Ști. Mat. Fiz. vol. 1 (1949) pp. 115-117.

18. N. Obrechkoff, Sur l'approximation des nombres irrationnels par des nombres rationnels, C. R. Acad. Bulgare Sci. vol. 3 (1950) pp. 1-4 (Russian).

19. C. D. Olds, Note on an asymmetric Diophantine approximation, Bull. Amer. Math. Soc. vol. 52 (1946) pp. 261-263.

20. Alexander Oppenheim, Rational approximations to irrationals, Bull. Amer. Math. Soc. vol. 47 (1941) pp. 602-604.

21. A. V. Prasad, Note on a theorem of Hurwitz, J. London Math. Soc. vol. 23 (1948) pp. 169-171.

22. R. M. Robinson, The approximation of irrational numbers by fractions with odd or even terms, Duke Math. J. vol. 7 (1940) pp. 354-359.

23. - Unsymmetrical approximation of irrational numbers, Bull. Amer. Math. Soc. vol. 53 (1947) pp. 351-361.

24. W. T. Scott, Approximation to real irrationals by certain classes of rational fractions, Bull. Amer. Math. Soc. vol. 46 (1940) pp. 124-129.

25. B. Segre, Lattice points in infinite domains and asymmetric Diophantine approximations, Duke Math. J. vol. 12 (1945) pp. 337-365.

26. V. T. S6s, On the theory of Diophantine approximations. II. Inhomogeneous problems, Acta Math. Acad. Sci. Hungar. vol. 9 (1958) pp. 229-241.

27. Leonard Tornheim, Asymmetric minima of quadratic forms and asymmetric Diophantine approximation, Duke Math. J. vol. 22 (1955) pp. 287-294.

UNIVERSITY OF OREGON,

EUGENE, OREGON

The UNIVERSITY OF MichigAN,

AnN Arbor, Michigan 